\title{
Predictors for hepatocellular carcinoma recurrence after microwave ablation
}

\author{
Hend Naguib Abd El Moteleub', Amr Ali Abd El Moety', Nahed Mohammed Baddour², Assem Ahmed El Shendidi \\ ${ }^{1}$ Hepatology Unit, Internal Medicine Department, Faculty of Medicine, Alexandria University, Egypt \\ 2Pathology Department, Faculty of Medicine, Alexandria University, Egypt
}

\begin{abstract}
Aim of the study: Microwave ablation (MWA) for treatment of hepatocellular carcinoma (HCC) is a new promising modality. The prognosis after treatment is mainly linked to the recurrence. We aimed to investigate the predictive value of $\alpha$-fetoprotein (AFP) score and Aurora B kinase (AURKB) in HCC recurrence after MWA.

Material and methods: A cross-sectional study where 25 early-stage HCC patients (Barcelona Clinic Liver Cancer O/A-B) were treated with MWA. Tumor biopsies were obtained just prior to MWA and assessed for WHO pathological grade and AURKB expression by immunohistochemistry. AFP score was calculated and a cut-off value of 2 classifies patients into high and low risk of recurrence. After achieving complete ablation, patients were followed every 3 months for 1 year by triphasic $\mathrm{CT}$ to detect recurrence.

Results: Child-Pugh classification has no significant impact on prognosis of HCC after MWA $\left(\chi^{2}=1.924\right.$, $p=0.165)$. Serum AFP level and AFP score can effectively predict the response to MWA among HCC patients $\left(\chi^{2}=6.451,{ }^{M C} p=0.031\right)\left(\chi^{2}=9.0, p=0.003\right)$, respectively. AFP score was strongly associated with the pathological grade of the tumor $(r=0.467, p=0.019)$. AURKB was over-expressed in tumoral more than non-tumoral specimens $(p<0.001)$. It was correlated with the size of the tumor, the number of tumor nodules and the pathological grade of the tumor $(p<0.05)$ but has no role in predicting recurrence after MWA $(p=0.869)$.

Conclusions: AFP score but not AURKB can predict the risk of recurrence of HCC after MWA.
\end{abstract}

Key words: recurrence, hepatocellular carcinoma, microwave ablation, AFP score, Aurora B kinase.

\section{Address for correspondence}

Dr. Hend Naguib Abd El Moteleub, Assistant Lecturer of Internal Medicine and Hepatology, Hepatology Unit, Internal Medicine Department, Faculty of Medicine, Alexandria University, 1 Khartoum Square, 21500, Alexandria, Egypt, e-mail: hendnaguib88@yahoo.com

\section{Introduction}

Hepatocellular carcinoma (HCC) is an international health problem with annual diagnosis of about 854,000 new cases [1]. It has been projected that by 2030 , this number will increase by $53 \%$ [2]. There are multiple choices for the treatment of HCC including surgical resection, liver transplantation, trans-arterial chemoembolization and percutaneous local ablation [3]. Microwave ablation (MWA) is one form of local thermal ablation which is a new promising technique for HCC treatment. It depends on production of electromagnetic waves which force dipole water molecules to continuously rotate billions of times per second with the oscillating electric field typically at 900 to $2500 \mathrm{MHz}$ leading to production of heat [4]. MWA displays several theoretical advantages over radiofrequency ablation (RFA) including the reduced "heat sink" effect [5], less tissue charring during ablation, thus being efficient for achieving a larger ablation zone [6], and deeper and faster penetration with a short ablation time [7]. The new version of MWA machines is equipped with a modern shaft which is provided by a cooling system to prevent overheating and protect the skin from burns. This addition increases the amount of energy delivered to the target tissue to facilitate 
creation of large ablation zones [8]. Eventually, larger lesions could be treated by two options, either by repositioning of the antennae [9] or through the use of multiple antennae simultaneously [10]. In fact, with the introduction of the new system, over $80 \%$ of complete ablation could be achieved in large HCC nodules (up to $8 \mathrm{~cm}$ in diameter) [11].

The high recurrence rate after HCC treatment is the main cause of the dismal prognosis [12]. The French Study Group for Liver Transplantation reported a new predictive model for detection of HCC recurrence: the AFP score, based on tumor size, number of nodules and AFP values [13]. The AFP has a curial role in tumor differentiation and vascular invasion. Its predictive value for detection of HCC recurrence is increased by combining it with the size and the number of the tumors $[13,14]$. The AFP score was found to be superior to Milan criteria in selection and predicting recurrence of HCC candidates for liver transplantation $[13,15]$.

Aurora B kinase is considered a member of the serine/threonine kinase family which is fundamental for accurate genomic stability [16]. AURKB is crucial for correct chromosomal alignment and segregation [17]. Aurora B kinase deletion will lead to chromosomal segregation failure, which is the cause of genomic instability and tumorigenesis [18]. There are lots of human cancers which have shown high AURKB expression and it is significantly correlated with tumor aggressiveness [19]. Recent studies reported that its up-regulation is significantly linked to the invasion of the portal vein and/or hepatic veins $[20,21]$. It was noted that its aberrant expression in HCC has a predictive value in HCC recurrence after curative hepatectomy [22]. To our knowledge, there is no study which reported the prognostic importance of AFP score and AURKB in HCC patients treated with microwave ablation. Thus, we aimed to investigate the clinical and prognostic significance of AFP score and AURKB in HCC patients managed by MWA.

\section{Material and methods}

A cross-sectional study, purposive sample where twenty-five patients with definite HCC on top of liver cirrhosis related to HCV who were referred to the Alexandria University Hepatobiliary Unit were included. A triphasic computed tomography (CT) abdomen and/or dynamic magnetic resonance imaging (MRI) were performed to confirm the diagnosis of HCC, determine the site, size and numbers of the tumors and to rule out invasion of the portal vein and hepatic veins. Inclusion criteria were early-stage HCC according to
Barcelona Clinic Liver Cancer (BCLC 0/A-B), which includes either single nodule not greater than $5 \mathrm{~cm}$, or up to three nodules with the largest of $3 \mathrm{~cm}$ in maximum diameter. Child $\mathrm{C}$ patients, patients with portal venous thrombosis or distant metastasis, or who received other treatment technique before MWA were excluded. All patients were subjected to clinical examination. Severity of liver disease was assessed by the modified Child-Pugh classification. Alfa-fetoprotein (AFP) was assessed by enzymelinked immunosorbent assay. A simplified AFP score version was used for calculation of the AFP score [13] with classification of patients according to tumor size $(<3 \mathrm{~cm}, 3-6 \mathrm{~cm}$ and $>6 \mathrm{~cm}$ ) and according to number of nodules (1-3 and $\geq 4$ ) then patients were classified into high and low risk of recurrence based on a cutoff value of 2 .

All nodules were chosen for ablation by MWA using a 14 gauge AMICA probe and an AMICA $2.45 \mathrm{GHz}$ generator (Italy). Wattage and duration used for ablation were chosen based on the manufacturer's guides. Biopsies from the tumor and the surrounding tissues were obtained just prior to MWA.

Aurora B kinase expression was assessed by immunohistochemistry of the tumor and the surrounding cirrhotic tissue according to streptavidin peroxidase technique using ab2254 (1:250; Abcam, USA) antibody. Aurora B kinase expression was observed in the form of brown nuclear staining in the cellular population in all cases stained with the antibody. A CH 20 BIMF 200, Olympus microscope (China) was used for quantification of Aurora B kinase under a magnification of $400 \times$. For pathological evaluation of each biopsy, $81 \times 12$ points (number of intersections in the grid used) were performed. All positively and negatively stained cells falling on the grid's intersections were counted and the mean score per biopsy was calculated. The positively stained points were expressed as percentage out of the total number of counted points. HCC grade was assessed according to the World Health Organization grading system [23].

\section{Follow-up}

A triphasic CT was performed 4 weeks (to give time for post-ablation edema, which may give a false impression of recurrence, to resolve) after the MWA to assess the local response. Patients with residual tumor activity were retreated with MWA. Another follow-up triphasic CT was performed after 4 weeks. All patients were followed by performing triphasic CT every 3 months after complete ablation for 1 year to detect any recurrence of HCC. 


\section{Ethical statement}

The study was performed in alignment with the revised Declaration of Helsinki (2013), and with Good Clinical Practice guidelines. Our study was approved by the Ethical Committee of the Faculty of Medicine, Alexandria University (IRB No. 00007555). Informed consent from all subjects included in the study was obtained.

\section{Statistical analysis}

Analysis was done using SPSS 20.0. Qualitative data were expressed as number and percentage. Quantitative data were expressed as range and mean \pm standard deviation. $P$ value less than $5 \%$ level is considered significant. The chi-square test was used to compare between different categorical variables. Fisher's exact or Monte Carlo correction for $\chi^{2}$ was used when more than $20 \%$ of the cells had an expected count less than 5 . Student's $t$-test was used to compare between two studied groups of normally distributed quantitative variables. The Mann-Whitney test was used to compare between two studied groups with non-normally distributed quantitative variables. The Pearson coefficient test was used for correlation between two normally distributed quantitative variables. Comparison between more than two studied groups with abnormally distributed quantitative variables was done using the Kruskal-Wallis test. Correlation between two abnormally distributed quantitative variables was done using the Spearman coefficient test.

\section{Results}

\section{Demographic data}

There was a male predominance in our studied sample: $19 / 25(76.0 \%)$ were male with a mean age of $56.13 \pm 6.77$ years, range (43.0-70.0) years.

\section{Clinico-pathological characteristics of studied cases}

Descriptive analysis of the studied cases according to clinico-pathological characteristics is illustrated in Table 1.

\section{Response to MWA therapy}

There was no significant difference between patients who achieved complete ablation and those who showed recurrence regarding Child-Pugh classes $\left(\chi^{2}=1.924\right.$, $p=0.165)$, tumor size $\left(\chi^{2}=4.081,{ }^{\mathrm{MC}} p=0.105\right)$, the
Table 1. Descriptive analysis of the studied cases according to clinicopathological characteristics

\begin{tabular}{|c|c|c|}
\hline Parameter & $n$ & $\%$ \\
\hline \multicolumn{3}{|l|}{ AFP level (ng/ml) } \\
\hline$\leqslant 100$ & 16 & 64.0 \\
\hline$>100-\leqslant 1000$ & 7 & 28.0 \\
\hline$>1000$ & 2 & 8.0 \\
\hline Min.-Max. & \multicolumn{2}{|c|}{$2.40-1132.0$} \\
\hline Mean $\pm S D$ & \multicolumn{2}{|c|}{$194.8 \pm 330.3$} \\
\hline Median (IQR) & \multicolumn{2}{|c|}{$41.80(8.1-178.9)$} \\
\hline \multicolumn{3}{|l|}{ Child class } \\
\hline$A$ & 12 & 48.0 \\
\hline B & 13 & 52.0 \\
\hline \multicolumn{3}{|l|}{ Tumor number } \\
\hline $1-3$ & 23 & 92.0 \\
\hline$\geqslant 4$ & 2 & 8.0 \\
\hline Min.-Max. & \multicolumn{2}{|c|}{$1.0-4.0$} \\
\hline Mean $\pm S D$ & \multicolumn{2}{|c|}{$1.72 \pm 1.02$} \\
\hline Median (IQR) & \multicolumn{2}{|c|}{$1.0(1.0-2.5)$} \\
\hline \multicolumn{3}{|l|}{ Tumor size (cm) } \\
\hline$\leqslant 3$ & 4 & 16.0 \\
\hline$<3-6$ & 18 & 72.0 \\
\hline$>6$ & 3 & 12.0 \\
\hline Min.-Max. & \multicolumn{2}{|c|}{$2.0-7.50$} \\
\hline Mean \pm SD & \multicolumn{2}{|c|}{$4.44 \pm 1.40$} \\
\hline Median (IQR) & \multicolumn{2}{|c|}{$4.20(3.5-5.2)$} \\
\hline \multicolumn{3}{|l|}{ Pathologic grade } \\
\hline$\|$ & 12 & 48.0 \\
\hline III & 13 & 52.0 \\
\hline \multicolumn{3}{|l|}{ AFP score } \\
\hline$\leqslant 2$ & 13 & 52.0 \\
\hline$>2$ & 12 & 48.0 \\
\hline Min.-Max. & \multicolumn{2}{|c|}{$0.0-6.0$} \\
\hline Mean $\pm S D$ & \multicolumn{2}{|c|}{$2.16 \pm 1.57$} \\
\hline Median (IQR) & \multicolumn{2}{|c|}{$2.0(1.0-3.0)$} \\
\hline
\end{tabular}

number of tumor nodules $\left(\chi^{2}=0.032,{ }^{\mathrm{FE}} p=1.000\right)$ and the pathological grade of the tumor $\left(\chi^{2}=0.051\right.$, $p=0.821)$. Serum AFP level and AFP score showed a statistically significant difference between patients who achieved complete ablation and patients with recurrence $\left(\chi^{2}=6.451,{ }^{\mathrm{MC}} p=0.031\right)\left(\chi^{2}=9.0, p=0.003\right)$, respectively. $78.6 \%$ of patients who achieved complete ablation after MWA were low-risk patients (AFP score $\leq 2) .81 .8 \%$ of patients who showed recurrence were high-risk patients (AFP score $>2$ ) (Table 2). 
Table 2. Relation between response to MWA therapy and different parameters

\begin{tabular}{|c|c|c|c|c|c|c|}
\hline & \multicolumn{4}{|c|}{ Response to MWA therapy } & \multirow[t]{3}{*}{ Test of sig. } & \multirow[t]{3}{*}{$p$} \\
\hline & \multicolumn{2}{|c|}{$\begin{array}{l}\text { Recurrence } \\
(n=11)\end{array}$} & \multicolumn{2}{|c|}{$\begin{array}{l}\text { Complete ablation } \\
\qquad(n=14)\end{array}$} & & \\
\hline & $N$ & $\%$ & $N$ & $\%$ & & \\
\hline \multicolumn{5}{|l|}{ Child class } & $\chi^{2}=1.924$ & 0.165 \\
\hline $\mathrm{A}$ & 7 & 63.6 & 5 & 35.7 & & \\
\hline$B$ & 4 & 36.4 & 9 & 64.3 & & \\
\hline \multicolumn{5}{|l|}{ Tumor size $(\mathrm{cm})$} & $\chi^{2}=4.081$ & ${ }^{M C} p=0.105$ \\
\hline$\leqslant 3$ & 1 & 9.1 & 3 & 21.4 & & \\
\hline$>3-6$ & 7 & 63.6 & 11 & 78.6 & & \\
\hline$>6$ & 3 & 27.3 & 0 & 0.0 & & \\
\hline Min.-Max. & \multicolumn{2}{|c|}{ 2.0-7.50 } & \multicolumn{2}{|c|}{$2.60-5.50$} & $t=1.393$ & 0.185 \\
\hline Mean $\pm S D$ & \multicolumn{2}{|c|}{$4.90 \pm 1.77$} & \multicolumn{2}{|c|}{$4.08 \pm 0.93$} & & \\
\hline Median & \multicolumn{2}{|c|}{4.50} & \multicolumn{2}{|c|}{4.05} & & \\
\hline \multicolumn{5}{|l|}{ AFP level (ng/ml) } & $\chi^{2}=6.451^{*}$ & ${ }^{M C} p=0.031^{*}$ \\
\hline$\leqslant 100$ & 4 & 36.4 & 12 & 85.7 & & \\
\hline$>100-\leqslant 1000$ & 5 & 45.5 & 2 & 14.3 & & \\
\hline$>1000$ & 2 & 18.2 & 0 & 0.0 & & \\
\hline \multicolumn{5}{|l|}{ Pathologic grade } & $\chi^{2}=0.051$ & 0.821 \\
\hline$\|$ & 5 & 45.5 & 7 & 50.0 & & \\
\hline III & 6 & 54.5 & 7 & 50.0 & & \\
\hline \multicolumn{5}{|l|}{ Tumor number } & $\chi^{2}=0.032$ & $\mathrm{FE} p=1.000$ \\
\hline $1-3$ & 10 & 90.9 & 13 & 92.9 & & \\
\hline$\geqslant 4$ & 1 & 9.1 & 1 & 7.1 & & \\
\hline \multicolumn{5}{|l|}{ AFP score } & $\chi^{2}=9.0^{*}$ & $0.003^{*}$ \\
\hline$\leqslant 2$ & 2 & 18.2 & 11 & 78.6 & & \\
\hline$>2$ & 9 & 81.8 & 3 & 21.4 & & \\
\hline Min.-Max. & \multicolumn{2}{|c|}{$1.0-6.0$} & \multicolumn{2}{|c|}{$0.0-3.0$} & $u=16.0^{*}$ & $<0.001^{*}$ \\
\hline Mean $\pm S D$ & \multicolumn{2}{|c|}{$3.36 \pm 1.29$} & \multicolumn{2}{|c|}{$1.21 \pm 1.05$} & & \\
\hline Median & \multicolumn{2}{|c|}{3.0} & \multicolumn{2}{|c|}{1.0} & & \\
\hline
\end{tabular}

$\chi^{2}$ - chi square test, MC - Monte Carlo, FE - Fisher exact test, $t$ - Student $t$-test, $U$ - Mann-Whitney test, *statistically significant at $p \leqslant 0.05$

\section{Comparison between high-risk (AFP score $>2$ ) and low-risk (AFP score $\leqslant 2$ ) patients regarding the pathological grade}

The high-risk and low-risk groups showed a statistically significant difference regarding the pathological grade of the tumor $\left(\chi^{2}=4.891, p=0.027\right)$ (Table 3$)$.

\section{Correlation between AFP score and pathological grade}

There was a significant positive correlation between AFP score and the pathological grade of the tumor $(r=0.467, p=0.019)$ (Table 4).

\section{Aurora B kinase expression in hepatocellular carcinoma and non-tumor liver tissue}

Aurora B kinase showed significantly high expression in HCC tumor tissue compared to corresponding non-tumor tissues $(p<0.001)$, with an average expression score of $63.56 \pm 17.64$ and $20.76 \pm 2.82$ respectively (Table 5).

Immunohistochemical staining of both tumoral and non-tumoral tissues showed that Aurora B kinase expression was higher in tumoral tissue than its non-tumoral counterparts (Fig. 1A, B). 
Table 3. Comparison between high-risk (AFP $>2$ ) and low-risk (AFP $\leqslant 2$ ) patients regarding the pathological grade

\begin{tabular}{|c|c|c|c|c|c|c|}
\hline \multirow{3}{*}{$\begin{array}{l}\text { Pathologic } \\
\text { grade }\end{array}$} & \multicolumn{4}{|c|}{ AFP score } & \multirow{3}{*}{$\begin{array}{l}\text { Test } \\
\text { of sig. }\end{array}$} & \multirow[t]{3}{*}{$p$} \\
\hline & \multicolumn{2}{|c|}{$\leqslant 2(n=13)$} & \multicolumn{2}{|c|}{$>2(n=12)$} & & \\
\hline & No. & $\%$ & No. & $\%$ & & \\
\hline II & 9 & 69.2 & 3 & 25.0 & $\chi^{2}=4.891^{*}$ & 0.027 \\
\hline III & 4 & 30.8 & 9 & 75.0 & & \\
\hline
\end{tabular}

Table 4. Correlation between AFP score with the pathological grade $(n=25)$

\begin{tabular}{lcc}
\hline & \multicolumn{3}{c}{ AFP score } \\
\cline { 2 - 3 } & $r_{s}$ & $p$ \\
\hline Pathologic grade & 0.467 & 0.019 \\
\hline
\end{tabular}

$r_{s}$-Spearman coefficient

Table 5. Aurora B kinase expression in HCC and non-tumoral liver tissue

\begin{tabular}{lcccc}
\hline & \multicolumn{2}{c}{ Aurora B kinase expression } & $Z$ & $p$ \\
\cline { 2 - 3 } & $\begin{array}{c}\text { Non-tumoral } \\
\text { liver tissue }\end{array}$ & $\begin{array}{c}\text { HCC } \\
\text { tissue }\end{array}$ & & \\
\hline Min.-Max. & $17.0-27.0$ & $39.0-89.0$ & \multirow{2}{*}{4.375} & $<0.001$ \\
\cline { 1 - 3 } Mean \pm SD & $20.76 \pm 2.82$ & $63.56 \pm 17.64$ & & \\
\hline Median (IQR) & 20.0 & 69.0 & & \\
\hline
\end{tabular}

$Z$ - Wilcoxon signed ranks test, $p$ - $p$-value for comparing between liver and tumor

\section{Aurora B kinase expression in HCC and its relation with different clinico-pathological parameters}

There was no significant relation between Aurora B kinase expression and sex of the patients. There was no significant difference in expression of Aurora B kinase between different groups of Child class, size of the tumor and serum AFP levels. However, a significant difference was observed between the scores of Aurora $B$ kinase expression in cases where the number of nodules was 3 or less and those with 4 or more tumors $(\mathrm{U}=2.0, p=0.035)$. The mean level of Aurora B kinase among the high-risk group was $71.58 \pm 16.49$ while in the low-risk group it was $56.15 \pm 15.78$. There was a statistically significant difference in the expression of Aurora B kinase between the two groups $(\mathrm{U}=41.50$, $p=0.047)$ (Table 6).

There was a statistically significant difference of Aurora B kinase expression between pathological grade II and pathological grade III $(\mathrm{U}=0.00, p<0.001)$ (Fig. 2A, B).

Regarding the prognostic significance of Aurora B kinase expression, there was no significant difference in Aurora B kinase expression between patients who
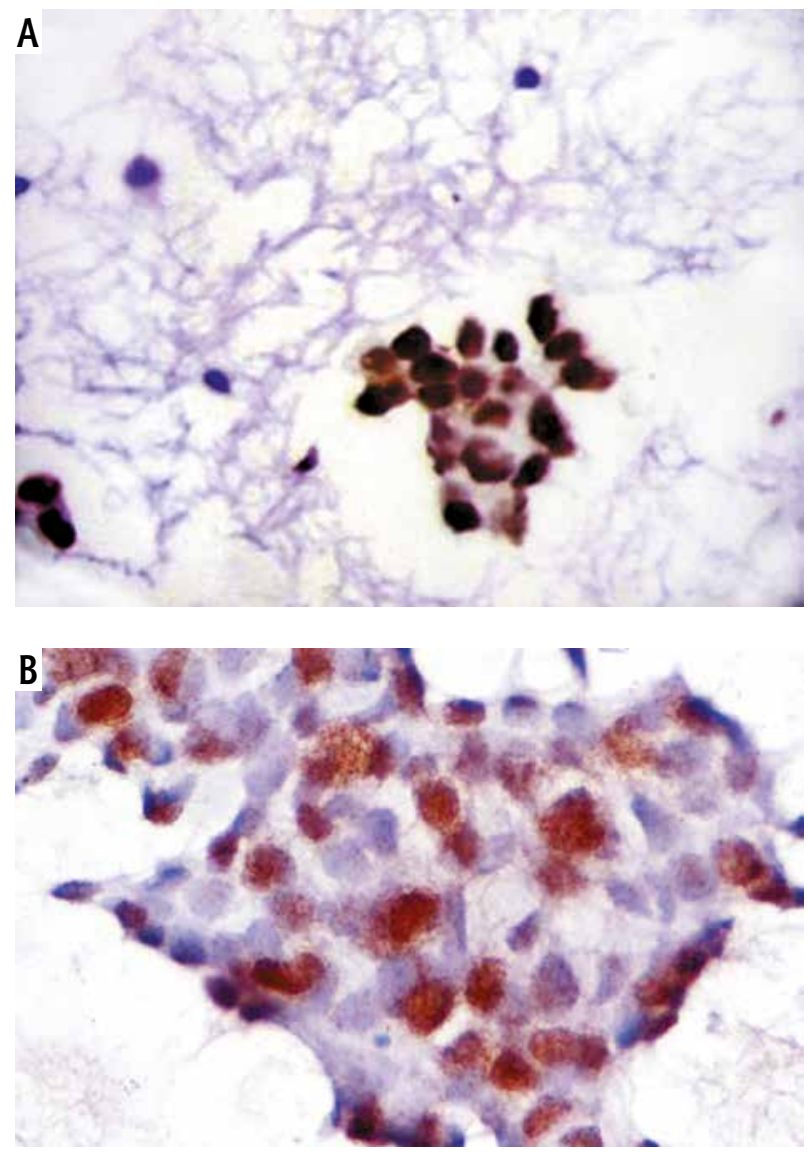

Fig. 1. Comparison of Aurora $B$ kinase expression between $\mathrm{HCC}$ tissue and non-tumoral liver tissue smears. A) Aurora B kinase staining in grade II HCC. Note the nuclear staining pattern (Aurora B kinase antibody, streptavidin peroxidase technique, 200x). B) Aurora B kinase antibody staining in nontumoral cirrhotic liver smears. Note the positive nuclear staining observed in several tumor cells seen in this cluster of reactive hepatocytes (Aurora B kinase antibody, streptavidin peroxidase technique, 400x)

achieved complete ablation and patients with recurrence $(p=0.869)$.

\section{Correlation between Aurora B kinase expression in HCC and different clinico-pathological parameters}

Statistically significant positive correlations were observed between the expression of Aurora B kinase on one hand and the size of the tumor, the number of tumor nodules, AFP score and the pathological grade of the tumor on the other hand. No correlation was found between Aurora B kinase score and Child class (Table 7).

\section{Discussion}

Microwave ablation is a new promising minimally invasive thermal modality for HCC treatment [24]. In spite of increasing numbers of patients who prefer microwave 
Table 6. Aurora B kinase expression in HCC and its relation with different clinico-pathological parameters

\begin{tabular}{|c|c|c|c|c|c|c|}
\hline \multirow[t]{2}{*}{ Parameters } & \multirow[t]{2}{*}{$n$} & \multicolumn{3}{|c|}{ Aurora B kinase expression in HCC } & \multirow[t]{2}{*}{ Test of sig. } & \multirow[t]{2}{*}{$p$} \\
\hline & & Min.-Max. & Mean $\pm S D$ & Median & & \\
\hline Sex & & & & & $U=48.50$ & 0.588 \\
\hline Male & 19 & $42.0-89.0$ & $64.32 \pm 17.94$ & 69.0 & & \\
\hline Female & 6 & $39.0-79.0$ & $61.17 \pm 18.05$ & 62.50 & & \\
\hline Child class & & & & & $U=75.0$ & 0.870 \\
\hline A & 12 & $39.0-89.0$ & $62.75 \pm 17.29$ & 61.50 & & \\
\hline$B$ & 13 & $43.0-88.0$ & $64.31 \pm 18.63$ & 73.0 & & \\
\hline Tumor number & & & & & $U=2.0^{*}$ & 0.035 \\
\hline $1-3$ & 23 & $39.0-89.0$ & $61.48 \pm 16.81$ & 54.0 & & \\
\hline$\geqslant 4$ & 2 & $87.0-88.0$ & $87.50 \pm 0.71$ & 87.50 & & \\
\hline Tumor size (cm) & & & & & $H=5.297$ & 0.071 \\
\hline$\leqslant 3$ & 4 & $43.0-54.0$ & $47.50 \pm 4.80$ & 46.50 & & \\
\hline$<3-6$ & 18 & $39.0-88.0$ & $64.17 \pm 17.69$ & 71.0 & & \\
\hline$>6$ & 3 & $76.0-89.0$ & $81.33 \pm 6.81$ & 79.0 & & \\
\hline AFP level (ng/ml) & & & & & $H=0.003$ & 0.999 \\
\hline$\leqslant 100$ & 16 & $39.0-89.0$ & $63.50 \pm 19.25$ & 62.0 & & \\
\hline$>100-\leqslant 1000$ & 7 & $42.0-79.0$ & $64.0 \pm 15.41$ & 69.0 & & \\
\hline$>1000$ & 2 & $47.0-78.0$ & $62.50 \pm 21.92$ & 62.50 & & \\
\hline AFP score & & & & & $U=41.50^{*}$ & 0.047 \\
\hline$\leqslant 2$ & 13 & $39.0-82.0$ & $56.15 \pm 15.78$ & 50.0 & & \\
\hline$>2$ & 12 & $42.0-89.0$ & $71.58 \pm 16.49$ & 77.50 & & \\
\hline Response to MWA therapy & & & & & $U=74.0$ & 0.869 \\
\hline Recurrence & 11 & $39.0-89.0$ & $64.45 \pm 18.61$ & 69.0 & & \\
\hline Complete ablation & 14 & $43.0-88.0$ & $62.86 \pm 17.51$ & 62.0 & & \\
\hline Pathologic grade & & & & & $U=0.00^{*}$ & $<0.001$ \\
\hline II & 12 & $39.0-54.0$ & $46.33 \pm 4.29$ & 46.0 & & \\
\hline III & 13 & $69.0-89.0$ & $79.46 \pm 5.88$ & 78.0 & & \\
\hline
\end{tabular}

$U$ - Mann-Whitney test, $H$ - H for Kruskal-Wallis test, $p$ - $p$-value for comparing between the studied categories

ablation with satisfactory complete ablation, recurrence can occur, which represents an important problem $[25,26]$.

Our study showed that Child-Pugh classification has no significant impact on prognosis of HCC after MWA. A similar observation was made by Wang et al. [24].

Our study showed the significant importance of serum AFP level in predicting HCC recurrence after MWA. Similar observations were also reported by Notarpaolo et al. [15], who concluded that there was a significant role of AFP level in the probability of HCC recurrence after liver transplantation. In contrast, Liang et al. [27], who studied the prognostic factors affecting the survival after MWA, reported that the pre-ablation serum AFP level was not related to prognosis.

It has been found in the present study that AFP score can effectively predict the response to MWA among HCC patients. Shaker et al. [28] conducted a study to investigate the validity of the use of the AFP score as a predictor of response in HCC patients after loco-regional treatment [radiofrequency ablation (RFA), transarterial chemoembolization (TACE)] and found that both RFA and TACE groups of patients with an AFP score less than or equal to 2 had a higher complete response rate and lower recurrence rate than patients with an AFP score more than 2. Similar observations were reported by Notarpaolo et al. [15], who found that the utility of the AFP score was better than the Milan criteria in selection of patients who were at low risk of recurrence, even if beyond Milan criteria. Thus, they stated that patients who were within Milan criteria, but had high AFP scores, should be paid special attention before considering them eligible for liver transplantation. Careful downstaging to AFP score $\leq 2$ in these patients by percutaneous ablation technique can be advised before transplantation with a fairly low risk of recurrence and excellent rate of 
survival. These expansions in the selection criteria to the AFP score for patients who were beyond the Milan criteria might result in a considerable increase of about $14 \%$ in the number of patients eligible for liver transplantation.

The present study showed a strong association between the AFP score and the pathological grade of the tumor. Similar results were obtained by Notarpaolo et al. [15].

In the present study, AURKB was over-expressed in tumoral more than non-tumoral specimens. Similar observations were reported by Tovuu et al. [29], who studied the expression of the Aurora B kinase gene in both HCC tumor and non-tumor liver tissues and explained its role in HCC. They found that the expression of Aurora B kinase in tumor tissues was significantly higher than in non-tumor tissues. These results go hand in hand with the findings of Yasen et al. [20] as it has a significant role in hepatocarcinogenesis.

In our study, Aurora B kinase expression was correlated with the size of the tumor, the number of tumor nodules and the pathological grade of the tumor. These results are consistent with those of Lin et al. [30], who reported that Aurora B kinase mRNA expression in HCC was correlated with the tumor size and the tumor grade. Similar results were obtained by Sistayanarain et al. [21], who concluded that Aurora-B kinase was over expressed in moderately to poorly differentiated HCC.

In our study we also found no significant difference of Aurora B kinase expression between different groups of AFP levels. In contrast, Lin et al. [30] reported that Aurora B overexpression was associated with high serum AFP level. In our study the lack of the correlation could be explained by the low serum level of AFP in most of our patients (AFP level $<100$ in $64 \%$ of patients and $>1000$ in only $8 \%$ ).

Our study demonstrated that Aurora B kinase expression was not associated with the reserve of liver function (Child-Pugh). Similar results were obtained by Lin et al. [30]. Contradictory results were reported by Tovuu et al. [29], who found that high Aurora B kinase gene expression showed a significant correlation with the severity of chronic liver disease.

Our study showed no relation between Aurora B kinase and HCC recurrence. Lin et al. [30] stated that Aurora B kinase overexpression was associated with early recurrence and poor prognosis of surgically resected HCC. Also, contradictory results were reported by Yasen et al. [20], who declared that Aurora B kinase was significantly overexpressed in HCC patients with vascular invasion (portal vein and/or hepatic veins), advanced stages and capsular invasion; hence, it was considered to be a marker for poor prognosis and recurrence.

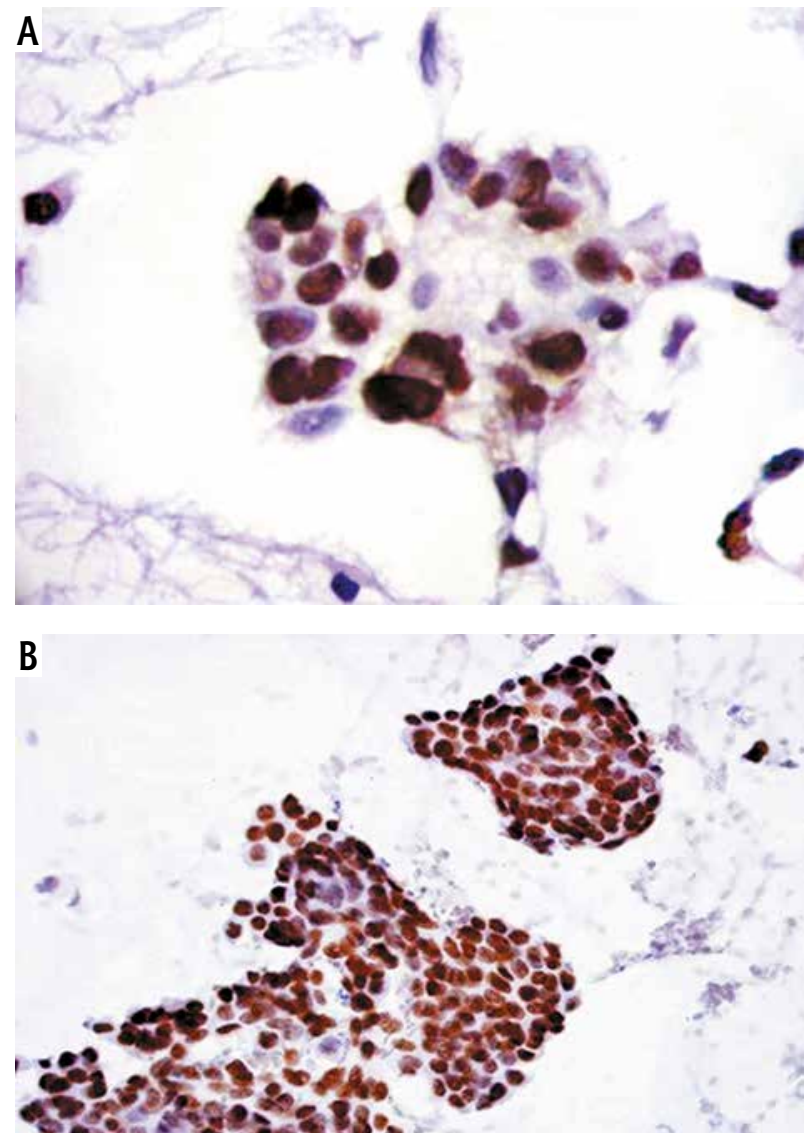

Fig. 2. Comparison of Aurora B kinase expression between grade II HCC and grade III HCC. A) Aurora B kinase staining in grade II HCC. Note the nuclear staining pattern (Aurora B kinase antibody, streptavidin peroxidase technique, 200x). B) Aurora B kinase staining in grade III HCC showing intense nuclear positivity (Aurora B kinase antibody, streptavidin peroxidase technique, 400x)

Table 7. Correlation between Aurora B kinase expression in HCC and different clinico-pathological parameters

\begin{tabular}{lcc}
\hline Parameters & \multicolumn{2}{c}{ Aurora in HCC (tumor) } \\
\cline { 2 - 3 } & $\boldsymbol{r}_{\mathrm{s}}$ & $\boldsymbol{p}$ \\
\hline Tumor size & $0.549^{*}$ & 0.004 \\
\hline Tumor number & $0.442^{*}$ & 0.027 \\
\hline AFP score & $0.471^{*}$ & 0.018 \\
\hline Pathologic grade & $0.867^{*}$ & $<0.001$ \\
\hline Child class & 0.033 & 0.874 \\
\hline
\end{tabular}

$r_{s}$ - Spearman coefficient

Since up-regulation of Aurora B kinase is significantly linked to the invasion of the portal vein and/or hepatic veins and high HCC stage and in turn to HCC recurrence $[20,21]$, the difference between our results and the results of other studies regarding the lack of correlation between Aurora B kinase expression in HCC and both Child class and recurrence of the tumor might be explained by the selection criteria in our study, which was limited to Child A and B, with exclusion of 
patients with Child $\mathrm{C}$, patients with vascular invasion and patients with extra-hepatic metastases.

\section{Conclusions}

In conclusion, it is evident that the AFP score not only serves as a score that could distinguish correctly and accurately between patients who are at low and high risk of HCC recurrence after microwave ablation but also can bring up more information about the tumor behavior, making the identification of aggressive tumors possible, even with reasonable tumor size and number. Aurora B kinase could be used as a biomarker for aggressiveness of HCC tumor (larger size, multinodularity and high grade) but not for prediction of HCC recurrence after microwave ablation.

We recommend conducting further studies with a larger sample size and longer followup period to study the predictive value of Aurora B kinase and AFP score for HCC recurrence after microwave ablation.

The main limitation of our study is the small sample size. The main strength is that it is a prospective study. Another strength is the novelty of the idea, since the role of AFP score and Aurora B kinase in HCC recurrence after microwave ablation has not been studied before.

\section{Disclosure}

The authors report no conflict of interest.

\section{References}

1. Addario L, Tritto G, Cavaglia E, et al. Preserved liver function, portal thrombosis and absence of oesophageal varices are risk factors for metastasis of hepatocellular carcinoma. Dig Liver Dis 2011; 43: 319-324.

2. Chaiteerakij R, Chattieng P, Choi J, et al. Surveillance for hepatocellular carcinoma reduces mortality: an inverse probability of treatment weighted analysis. Ann Hepatol 2017; 16: 421-429.

3. Beppu T, Sugimoto K, Shiraki K, et al. Clinical significance of tumor markers in detection of recurrent hepatocellular carcinoma after radiofrequency ablation. Int J Mol Med 2010; 26: 425-433.

4. European Association for the Study of the Liver. EASL Clinical Practice Guidelines: Management of hepatocellular carcinoma. J Hepatol 2018; 69: 182-236.

5. Lucchina N, Tsetis D, Ierardi AM, et al. Current role of microwave ablation in the treatment of small hepatocellular carcinomas. Ann Gastroenterol 2016; 29: 460-465.

6. Minami Y, Kudo M. Radiofrequency ablation of hepatocellular carcinoma: Current status. World J Radiol 2010; 2: 417-424.

7. Yu J, Liang P, Yu X, et al. A comparison of microwave ablation and bipolar radiofrequency ablation both with an internally cooled probe: results in ex vivo and in vivo porcine livers. Eur J Radiol 2011; 79: 124-130.

8. Liu Y, Zheng Y, Li S, et al. Percutaneous microwave ablation of larger hepatocellular carcinoma. Clin Radiol 2013; 68: 21-26.

9. Tang XY, Wang Z, Wang T, et al. Efficacy, safety and feasibility of ultrasound-guided percutaneous microwave ablation for large hepatic hemangioma. J Dig Dis 2015; 16: 525-530.
10. Forner A, Llovet JM, Bruix J. Hepatocellular carcinoma. Lancet 2012; 379: 1245-1255.

11. Zhang NN, Lu W, Cheng XJ, et al. High-powered microwave ablation of larger hepatocellular carcinoma: evaluation of recurrence rate and factors related to recurrence. Clin Radiol 2015; 70: 1237-1243.

12. Portolani N, Coniglio A, Ghidoni S, et al. Early and late recurrence after liver resection for hepatocellular carcinoma: prognostic and therapeutic implications. Ann Surg 2006; 243: 229-235.

13. Duvoux C, Roudot-Thoraval F, Decaens T, et al. Liver transplantation for hepatocellular carcinoma: a model including alpha-fetoprotein improves the performance of Milan criteria. Gastroenterology 2012; 143: 986-994.

14. Liu C, Xiao GQ, Yan LN, et al. Value of alpha-fetoprotein in association with clinicopathological features of hepatocellular carcinoma. World J Gastroenterol 2013; 19: 1811-1819.

15. Notarpaolo A, Layese R, Magistri P, et al. Validation of the AFP model as a predictor of HCC recurrence in patients with viral hepatitis-related cirrhosis who had received a liver transplant for HCC. J Hepatol 2017; 66: 552-559.

16. Keen N, Taylor S. Aurora-kinase inhibitors as anticancer agents. Nat Rev Cancer 2004; 4: 927-936.

17. Mita AC, Mita MM, Nawrocki ST, et al. Survivin: key regulator of mitosis and apoptosis and novel target for cancer therapeus. Clin Cancer Res 2008; 14: 5000-5005.

18. Torchia EC, Zhang L, Huebner AJ, et al. Aurora kinase-A deficiency during skin development impairs cell division and stratification. J Invest Dermatol 2013; 133: 78-86.

19. Zardavas D, Maetens M, Irrthum A, et al. The AURORA initiative for metastatic breast cancer. Br J Cancer 2014; 111: 1881-1887.

20. Yasen M, Mizushima H, Mogushi K, et al. Expression of Aurora $\mathrm{B}$ and alternative variant forms in hepatocellular carcinoma and adjacent tissue. Cancer Sci 2009; 100: 472-480.

21. Sistayanarain A, Tsuneyama K, Zheng H, et al. Expression of Aurora-B kinase and phosphorylated histone $\mathrm{H} 3$ in hepatocellular carcinoma. Anticancer Res 2006; 26: 3585-3593.

22. Tanaka S, Arii S, Yasen M, et al. Aurora kinase B is a predictive factor for the aggressive recurrence of hepatocellular carcinoma after curative hepatectomy. Br J Surg 2008; 95: 611-619.

23. Bosman FT, Carneiro F, Hruban RH, et al. WHO Classification of Tumours of the Digestive System. 4th ed. World Health Organization, Geneva, Switzerland 2010.

24. Wang ZL, Liang P, Dong BW, et al. Prognostic factors and recurrence of small hepatocellular carcinoma after hepatic resection or microwave ablation: a retrospective study. J Gastrointest Surg 2008; 12: 327-337.

25. Liang P, Wang Y. Microwave ablation of hepatocellular carcinoma. Oncology 2007; 72 Suppl 1: 124-131.

26. Liang P, Yu J, Yu XL, et al. Percutaneous cooled-tip microwave ablation under ultrasound guidance for primary liver cancer: a multicentre analysis of 1363 treatment-naive lesions in 1007 patients in China. Gut 2012; 61: 1100-1101.

27. Liang P, Dong B, Yu X, et al. Prognostic factors for survival in patients with hepatocellular carcinoma after percutaneous microwave ablation. Radiology 2005; 235: 299-307.

28. Shaker MK, Abdella HM. Validation of AFP model as a predictor of response, recurrence and survival in hcc patients underwent locoregional treatment. Egypt J Hosp Med 2018; 72: 5470-5478.

29. Tovuu LO, Utsunomiya T, Imura S, et al. The role of Aurora B expression in non-tumor liver tissues of patients with hepatocellular carcinoma. Int J Clin Oncol 2014; 19: 622-628.

30. Lin ZZ, Jeng YM, Hu FC, et al. Significance of Aurora B overexpression in hepatocellular carcinoma. Aurora B Overexpression in HCC. BMC Cancer 2010; 10: 461. 